\title{
Lifshitz-Slyozov kinetics of a nonconserved system that separates into phases of different density
}

Mouritsen, Ole G.; Shah, Peter Jivan; Andersen, Jørgen Vitting

\section{Published in:}

Physical Review B

Link to article, DOI:

10.1103/PhysRevB.42.4506

Publication date:

1990

Document Version

Publisher's PDF, also known as Version of record

Link back to DTU Orbit

Citation (APA):

Mouritsen, O. G., Shah, P. J., \& Andersen, J. V. (1990). Lifshitz-Slyozov kinetics of a nonconserved system that separates into phases of different density. Physical Review B, 42(7), 4506-4513.

https://doi.org/10.1103/PhysRevB.42.4506

\section{General rights}

Copyright and moral rights for the publications made accessible in the public portal are retained by the authors and/or other copyright owners and it is a condition of accessing publications that users recognise and abide by the legal requirements associated with these rights.

- Users may download and print one copy of any publication from the public portal for the purpose of private study or research.

- You may not further distribute the material or use it for any profit-making activity or commercial gain

- You may freely distribute the URL identifying the publication in the public portal 


\title{
Lifshitz-Slyozov kinetics of a nonconserved system that separates into phases of different density
}

\author{
Ole G. Mouritsen, Peter Jivan Shah, and J $\phi$ rgen Vitting Andersen \\ Department of Structural Properties of Materials, The Technical University of Denmark, Building 307, \\ DK-2800 Lyngby, Denmark
}

(Received 27 April 1990)

\begin{abstract}
Computer-simulation techniques are applied to analyze the late-stage ordering kinetics of a two-dimensional annealed dilute Ising model quenched into regions of its phase diagram that involve phase separation of phases with different densities. The order parameter of the model is a nonconserved quantity, whereas the global density is conserved. The ordered phases of the model are fourfold degenerate $(2 \times 1)$ and $(2 \times 2)$ superstructures on a square lattice. The equilibrium phase diagram involves a region of coexisting $(2 \times 1)$ and $(2 \times 2)$ phases and a region where the $(2 \times 2)$ phase coexists together with a gas phase. The results of the study show that the phase-separation kinetics in all cases are consistent with the Lifshitz-Slyozov growth law, $\bar{R}(t) \sim t^{1 / 3}$, where $\bar{R}(t)$ is the characteristic linear domain size. These results are in agreement with recent low-energy electron-diffraction studies of the phase separation kinetics in $\mathrm{O} / \mathrm{W}(110)$ systems at high coverage.
\end{abstract}

\section{INTRODUCTION}

The late-stage kinetics of nonequilibrium systems undergoing ordering processes, such as domain growth, spinodal decomposition, and phase separation, is strongly influenced by the conservation laws in effect. ${ }^{1-4}$ In particular, the conservation law for the order parameter is of paramount importance. In general it has been found, ${ }^{5}$ theoretically as well as experimentally, that Lifshitz-Allen-Cahn kinetics, $\bar{R}(t) \sim t^{1 / 2}$, follows when the order parameter is nonconserved (model $A$ of critical dynamics ${ }^{6}$ ), whereas the Lifshitz-Slyozov law, $\bar{R}(t) \sim$ $t^{1 / 3}$, applies when the order parameter is conserved ${ }^{7-19}$ (model $B$ ). $\bar{R}(t)$ is a measure of the average linear size of the ordering domains. A complication arises when the order parameter is not conserved but at the same time is coupled to some other quantity, such as the density that is conserved (model $C$ ). In that case it becomes of importance whether or not the involved domains and phases have the same density. If the densities are the same, e.g., as in the problem of domain growth in a model with simple order-parameter degeneracy, ${ }^{20,21}$ the Lifshitz-Allen-Cahn growth law applies. If, however, the densities of the involved phases are not the same, longrange-transport processes are needed to facilitate the separation process and the Lifshitz-Slyozov growth is again expected to apply. ${ }^{22}$ A carefully studied example of the latter case is that of a quenched tricritical system where the Lifshitz-Slyozov law has been found to indeed describe the late-stage-ordering kinetics. ${ }^{23-25}$ The LifshitzSlyozov theory ${ }^{26,27}$ was originally developed to describe spinodal decomposition at low volume fractions. However, it has recently been shown ${ }^{28,29}$ that the theory can be generalized to apply for arbitrary volume fractions of the two phases.

Chemisorbed molecules on solid surfaces constitute a class of systems ${ }^{3}$ that are prototypical for systems undergoing ordering processes in which the density (coverage) is conserved but the order parameter is not. These chemisorbed systems display ordered structures with sublattice ordering. Hence the lateral diffusion leads to nonconservation of the order parameter during the ordering process. Only for $(1 \times 1)$ structures, where the density and the order parameter is the same quantity, is the order parameter conserved. Chemisorbed systems display a very rich phase behavior with a variety of differently ordered phases and associated phase-coexistence regions. Hence this class of systems provides a rich laboratory for investigating the kinetics of domain growth and phase separation involving phases of different densities. Experimentally, this is a field that is just opening up ${ }^{30-34}$ due to the application of high-resolution time-resolved techniques.

In the present work we report on the results of a computer-simulation study of the ordering kinetics and phase-separation dynamics in a particular twodimensional site-diluted kinetic Ising model with antiferromagnetic interactions that has a phase diagram containing regions of coexisting phases of different density. The vacancies of the model are mobile and hence enter directly into the ordering process and the phase separation. We are here going to consider very dilute situations where about half of the lattice sites are unoccupied. The low-dilution limit of the same model was investigated recently in the context of a study of the effect of annealed randomness on domain growth kinetics. ${ }^{35,36}$

\section{MODEL}

The present theoretical study is built on a kinetic lattice model. A kinetic lattice model is defined by two 
properties: (a) a Hamiltonian that governs a phase transition associated with certain ordering symmetries, and (b) a kinetic or dynamic principle (a move class) that specifies the possible transitions of the model and the associated transition probabilities.

\section{A. Hamiltonian and order parameters}

The site-diluted square-lattice spin- $\frac{1}{2}$ Ising antiferromagnet is defined by the Hamiltonian

$$
\mathcal{H}=J\left(\sum_{i>j}^{\mathrm{NN}} \sigma_{i} \sigma_{j}+\sum_{i>j}^{\mathrm{NNN}} \sigma_{i} \sigma_{j}\right),
$$

where $\sigma_{i}=0, \pm 1$ and the two summations extend over nearest-neighbor (NN) and next-nearest-neighbor (NNN) interactions, respectively. The spin value $\sigma_{i}=0$ is associated with vacant sites and the total vacancy concentration is $c$. The interaction constant is chosen to be antiferromagnetic, $J>0$. The model is arrayed on a square lattice with open boundaries and $N=L \times L$ sites.

The ground state of the model in the nondiluted case $c=0$ is the fourfold-degenerate superantiferromagnetic $(2 \times 1)$ structure shown in Fig. 1(a). Dilution of the system releases the inherent frustration of the $\mathrm{NN}$ bonds in the $(2 \times 1)$ structure and hence tends to stabilize another superstructure in which the vacancies arrange themselves in a sublattice ordering as shown in Fig. 1(b) for $c=\frac{1}{2}$. This structure is a fourfold-degenerate $(2 \times 2)$ structure, which is in fact a $p(2 \times 2)$ structure.

If we decompose the square lattice into four equivalent square sublattices, $S_{1}, S_{2}, S_{3}$, and $S_{4}$, with the double lattice parameter as indicated in Fig. 1(a), we can define the various order parameters in terms of the sublattice magnetizations

$$
\eta_{i}=N^{-1} \sum_{j \in S_{i}} \sigma_{j}
$$

The four components of the $(2 \times 1)$ order parameter are

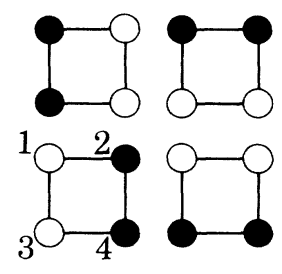

(a)

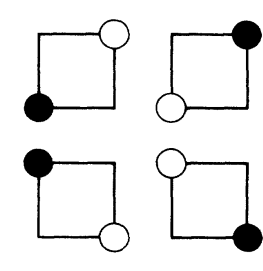

(b)
FIG. 1. Ground-state structures for the spin- $\frac{1}{2}$ Ising model in Eq. (1). (a) Fourfold-degenerate $(2 \times 1)$ ordering for $c=0$. The labeling 1-4 refers to the four sublattices used for defining the order parameters in Eqs. (3)-(12). (b) Fourfolddegenerate $(2 \times 2)$ ordering for $c=\frac{1}{2}$. Solid and open circles denote spin up and spin down, respectively. then given by

$$
\begin{aligned}
& \psi_{1}=\eta_{1}-\eta_{2}+\eta_{3}-\eta_{4}, \\
& \psi_{2}=-\eta_{1}+\eta_{2}-\eta_{3}+\eta_{4} \\
& \psi_{3}=-\eta_{1}-\eta_{2}+\eta_{3}+\eta_{4} \\
& \psi_{4}=\eta_{1}+\eta_{2}-\eta_{3}-\eta_{4},
\end{aligned}
$$

and the four components of the $(2 \times 2)$ order parameter as

$$
\begin{aligned}
& \phi_{1}=2\left(\eta_{3}-\eta_{2}\right), \\
& \phi_{2}=2\left(\eta_{2}-\eta_{3}\right), \\
& \phi_{3}=2\left(\eta_{1}-\eta_{4}\right), \\
& \phi_{4}=2\left(\eta_{4}-\eta_{1}\right) .
\end{aligned}
$$

Furthermore, a two-component vacancy order parameter can be defined as

$$
\begin{aligned}
& \phi_{1}^{v}=2 \sum_{j \in S_{1}, S_{4}} \delta_{\sigma_{j}}, \\
& \phi_{2}^{v}=2 \sum_{j \in S_{2}, S_{3}} \delta_{\sigma_{j}},
\end{aligned}
$$

where $\delta_{\sigma_{j}}=1$ for $\sigma_{j}=0$ and $\delta_{\sigma_{3}}=0$ otherwise. The various order parameters defined above are not independent. The symmetry properties of the $(2 \times 1)$ structure are realized physically for half-monolayer coverages of oxygen on $W(110)$ surfaces. ${ }^{34}$

\section{B. Model dynamics}

The Ising model does not have a natural dynamics of its own. We therefore associate it with a microscopic stochastic dynamic principle that involves singlesite spin-flip excitations as well as spin-vacancy exchange (translational diffusion). The spin-vacancy exchange involves exchange of a spin and a vacancy at NN or NNN positions as well as an attempt to simultaneously flip the spin involved in the exchange. This dynamical principle does not conserve any of the order parameters in Eqs. (3)-(12), but it conserves the vacancy concentration, i.e., the density.

The dynamic principle is expressed in a standard Monte Carlo Metropolis scheme. A site, $i$, and a $\mathrm{NN}$ or NNN site, $j$, to $i$ are chosen at random and the following possibilities are considered: (i) If $\sigma_{i}=\sigma_{j}=0$, nothing happens. (ii) If $\sigma_{i} \neq 0$ and $\sigma_{j} \neq 0, \sigma_{i}$ is randomly assigned a new value \pm 1 (spin flip) with the standard Metropolis-transition probability of a spin flip. (iii) If $\sigma_{i} \neq 0$ and $\sigma_{j}=0$, or $\sigma_{i}=0$ and $\sigma_{j} \neq 0$, the combined process of exchanging the site variables and randomly assigning a value \pm 1 to the site carrying the spin is performed according to the Metropolis-transition 
probability of the combined process. In any of the three cases (i)-(iii), the time parameter $t$ is incremented by one unit. This corresponds to a particularly simple choice of time-scale ratio for the spin-flip dynamics and the vacancy diffusivity. The time is measured in units of Monte Carlo steps per site (MCS/s). We have used a previously developed ${ }^{36}$ implementation of this updating scheme for a vector processor. The implementation is based on a generalized checkerboard updating principle according to which the lattice is subdivided into 16 equivalent interpenetrating square sublattices. By this very open decomposition it is possible to circumvent problems that will arise in a tighter decomposition where the exchange mechanism will correlate the sublattices. In the chosen open decomposition the 16 sublattices are independent and can be updated simultaneously. The consequences of a vectorized dynamics for the scaling of the time parameter were investigated in a previous paper in which it was found ${ }^{36}$ that the degree of vectorization does not influence the Markov time parameter. This result is of importance when the nonequilibrium properties are analyzed in terms of growth laws and dynamical scaling.

\section{PHASE DIAGRAM}

The equilibrium phase diagram has been determined by Monte Carlo equilibrium simulations, ${ }^{36}$ Fig. 2 . Since our main use of the phase diagram is to guide the nonequilibrium quenching simulations into coexistence regions, we have not attempted to obtain either a very accurate determination of the phase boundaries or a clarification of the nature of the transitions near the multicritical point. At low temperatures the phase diagram

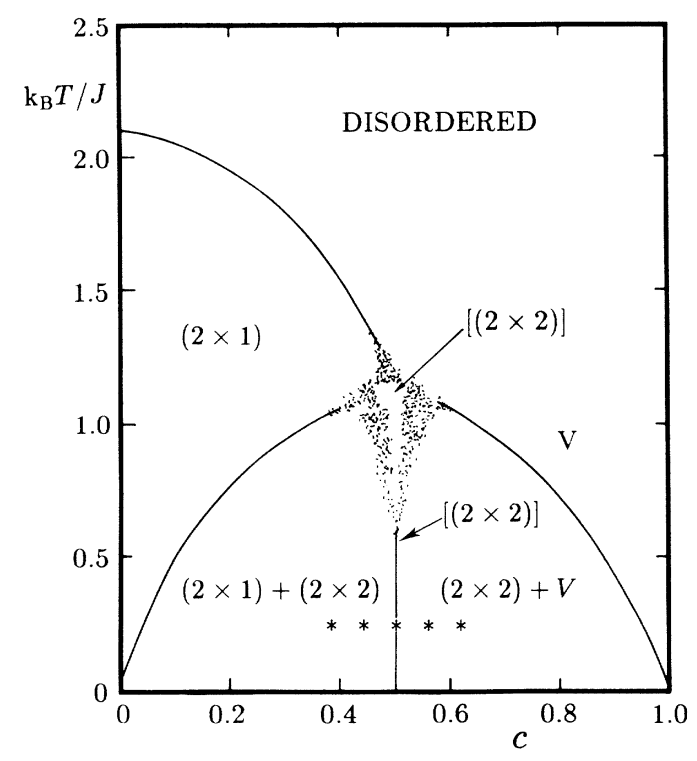

FIG. 2. Phase diagram of the model in Eq. (1) as determined by Monte Carlo simulations. The shaded areas denote regions of the phase diagram where the numerical determination of the phase boundaries is uncertain. The symbols $*$ indicate the points to which quenches have been performed. contains two regions of phase coexistence: Below $c=\frac{1}{2}$ the system separates into a diluted $(2 \times 1)$ phase and a $(2 \times 2)$ phase with excess density; above $c=\frac{1}{2}$ the system separates into a diluted $(2 \times 2)$ phase and an almost pure vacancy phase (a gas phase). At low temperatures the stability region for a homogeneous $(2 \times 2)$ phase near $c=\frac{1}{2}$ is very small and cannot, within the numerical uncertainty, be distinguished from a single line, i.e., the $(2 \times 2)$ phase can effectively be considered a so-called stoichiometric compound.

\section{ORDERING AND PHASE SEPARATION KINETICS}

\section{A. Quenching and measures of growth}

We are now going to consider thermal quenches, for fixed density $c$, from infinite temperature to temperatures well inside the ordered phases of the phase diagram. The major part of our results refers to quenches to a temperature $k_{B} T / J=0.25$. We have also performed a few selected quenches to $k_{B} T / J=0.50$ and 0.1 and have confirmed that the resulting growth characteristics do not depend on temperature. We have considered two densities, $c=0.40$ and 0.45 , in the $(2 \times 1)-(2 \times 2)$ coexistence region, two densities, $c=0.55$ and 0.60 , in the $(2 \times 2)$-gas coexistence region, as well as the stoichiometric density $c=0.50$. Each type of quench is carried out in ten different realizations using different initial (random) configurations and different random-number sequences for the Monte Carlo Metropolis algorithm. The results are averaged over these different realizations in order to obtain ensemble averages. This type of averaging is particularly important when non-self-averaging quantities ${ }^{\mathbf{3 7}}$ are considered.

The time evolution of the ordering processes and the phase separation is monitored by calculating a number of measures of linear length scale. One of these is the inverse of the excess energy per site,

$$
\Delta E(t)=E(t)-E(T)
$$

where $E(t)$ is the nonequilibrium energy and $E(T)=$ $E(t \rightarrow \infty)$ is the equilibrium energy. $\Delta E(t)$ is a measure of the internal energy associated with the entire nonequilibrium domain-boundary network. If scaling holds, ${ }^{38}$ $\Delta E^{-1}(t)$ is a measure of the average linear domain size. A more direct measure of the average domain size, $\bar{R}(t)$, can be calculated from the full domain-size distribution function, $P(R, t)$, which we have also calculated. A further measure of the growing ordered domains can be obtained from the order parameters, ${ }^{21}$ specifically

$$
\begin{aligned}
& L_{\psi}(t)=\sqrt{N}\left(\sum_{i=1}^{4}\left[\psi_{i}(t)\right]^{2}\right), \\
& L_{\phi}(t)=\sqrt{N}\left(\sum_{i=1}^{4}\left[\phi_{i}(t)\right]^{2}\right)^{1 / 2},
\end{aligned}
$$


and

$$
L_{v}(t)=\sqrt{N}\left(\sum_{i=1}^{2}\left[\phi_{i}^{v}(t)\right]^{2}\right)^{1 / 2} .
$$

Since neither of these three length-scale measures, which are related to the intensity of the scattering function at the Bragg point, are self-averaging quantities, they are more difficult to determine accurately than $\Delta E^{-1}(t)$ and $\bar{R}(t)$. This is particularly true of $L_{v}(t)$, which refers to only a twofold-degenerate ordering.

\section{B. Kinetics, growth laws, and dynamical scaling}

In Fig. 3 are shown some typical snapshots of microconfigurations as they evolve in time for $c=0.40,0.50$, and 0.60 . These snapshots illustrate the coarsening and phase-separation process and how it qualitatively depends on the vacancy concentration. At $c=0.40$ the majority phase is the $(2 \times 2)$ ordering with the $(2 \times 1)$ domains separated out as small clusters. The domain boundaries between the different $(2 \times 2)$ domains are seen as defect lines with either excess spins (dark lines) or excess vacancies (white lines). Hence there are heavy as well as light domain walls. There is some tendency for accumulation of vacancies in the domain boundaries. At $c=0.60$ there are only traces left of the $(2 \times 1)$ phase, and the vacancy phase appears as a thick-boundary network separating the $(2 \times 2)$ domains. At $c=0.50$, the $(2 \times 2)$ domains have grown to much larger sizes than they have at the corresponding times for higher and lower concentrations. Furthermore, these domains appear more compact than the convoluted (percolative) domain patterns at $c=0.40$ and 0.60 . There is a strong tendency for accumulation of vacancies in the domain boundaries. It is further noted that there is a transient precipitation of small $(2 \times 1)$ droplets that compensate for the low density of the domain walls.

The kinetics of the ordering and phase separation process is analyzed in Figs. 4-7 where the various lengthscale measures are plotted versus time in double logarithmic plots. The major result derived from these sets of data is that the ordering process proceeds in an algebraic fashion for all investigated values of $c$. Moreover, all measures of the growth give rise to the same growth
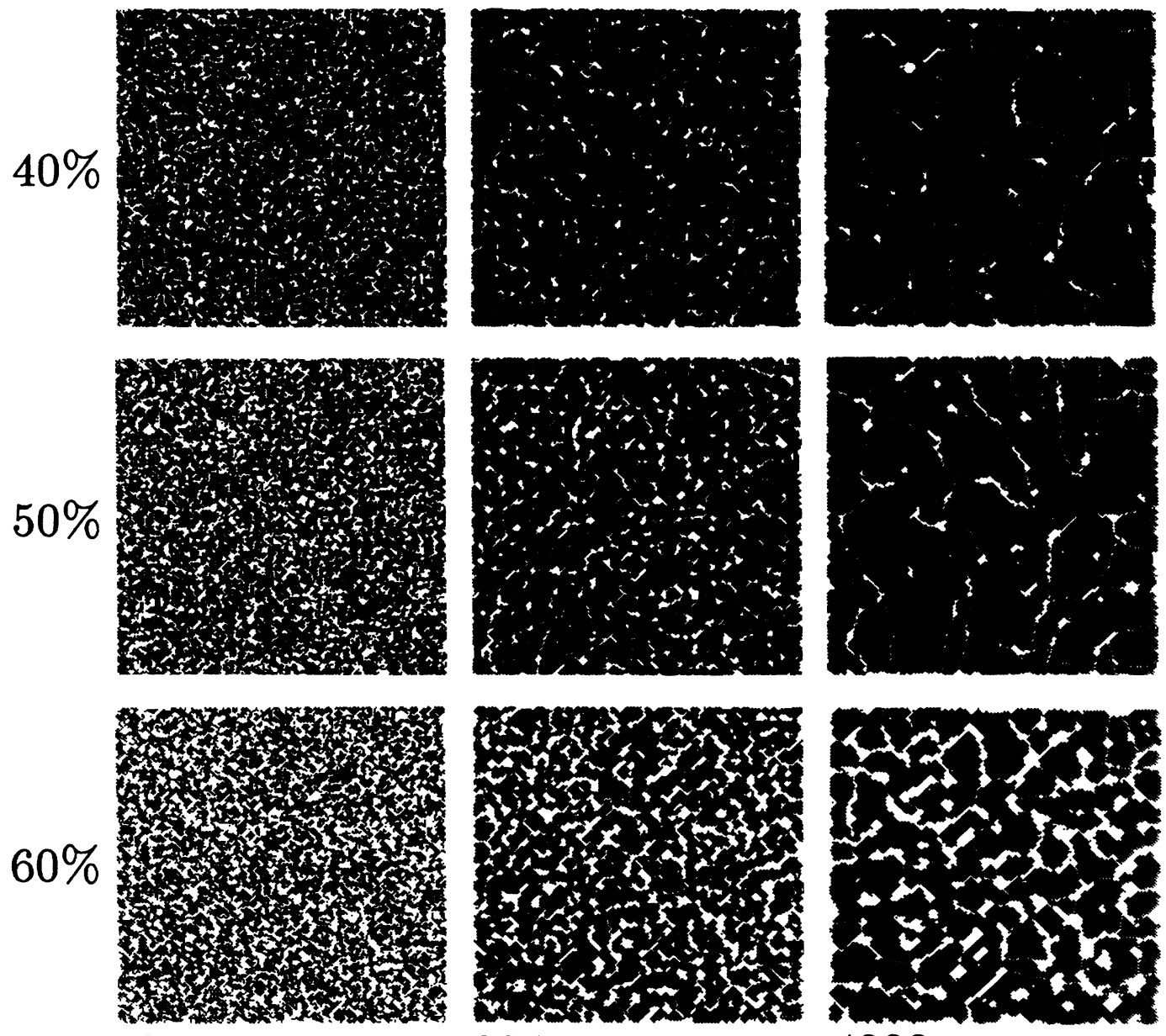

$\mathrm{t}=16$

234
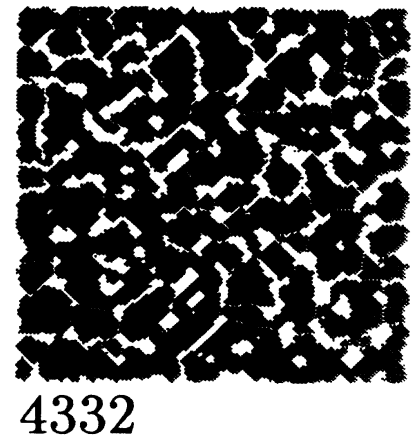

FIG. 3. Snapshots of typical microconfigurations at three different concentrations as they evolve in time $t$ (in units of $\mathrm{MCS} / \mathrm{s})$ after quenches to a temperature $k_{B} T / J=0.25$. Vacancies are denoted by blanks, $(2 \times 1)$ ordering is indicated in black, and $(2 \times 2)$ ordering appears as grey. 


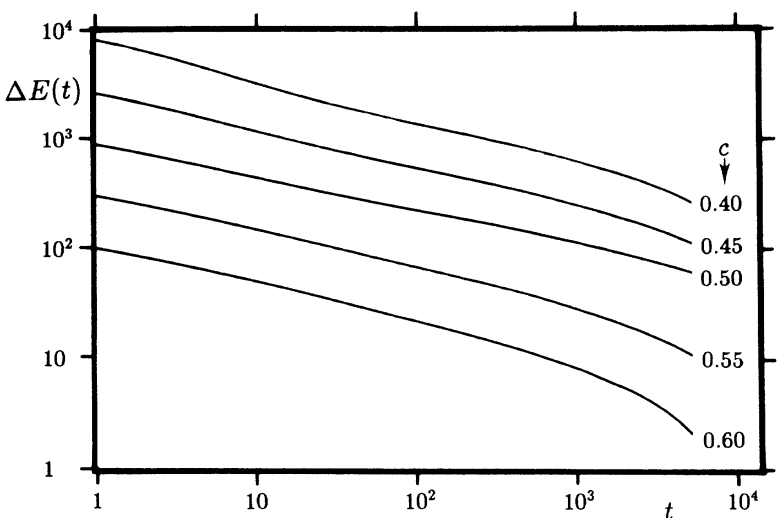

FIG. 4. Log-log plot of the excess energy $\Delta E(t)$, Eq. (13), vs time (in units of MCS/s) for five different vacancy concentrations $c$. The data, which for the sake of clarity have been appropriately translated along the vertical axis, refer to Monte Carlo simulations of systems with $200 \times 200$ sites quenched to a temperature $k_{B} T / J=0.25$; cf. the phase diagram in Fig. 2.

law

$$
\Delta E^{-1}(t) \sim \bar{R}(t) \sim L_{\phi}(t) \sim L_{v}(t) \sim t^{n}
$$

with $n \simeq 0.30 \pm 0.05$, and scaling is therefore obeyed. The algebraic growth law is found to apply for almost three decades in time. There is a trend in the data that shows that the effective exponent value becomes slightly lower as $c$ is decreased.

Due to previously mentioned problems with the lack of self-averaging, the quantity $L_{v}(t)$ shows considerably more scatter than the other length-scale measures. Nevertheless, the $L_{v}(t)$ data conform to the same growth law, Eq. (17).

The dynamical-scaling properties of the ordering process are investigated in more detail in Fig. 8, which shows a plot of the linear-domain-size distribution function, $P(R, t)$, in the case of $c=0.60$ for $(2 \times 2)$ domains. This figure demonstrates that $P(R, t)$ develops a peak

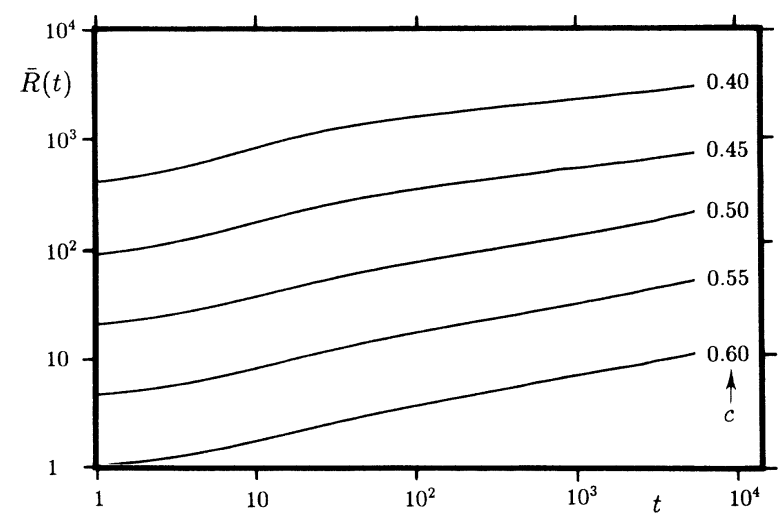
$\bar{R}(t)$.

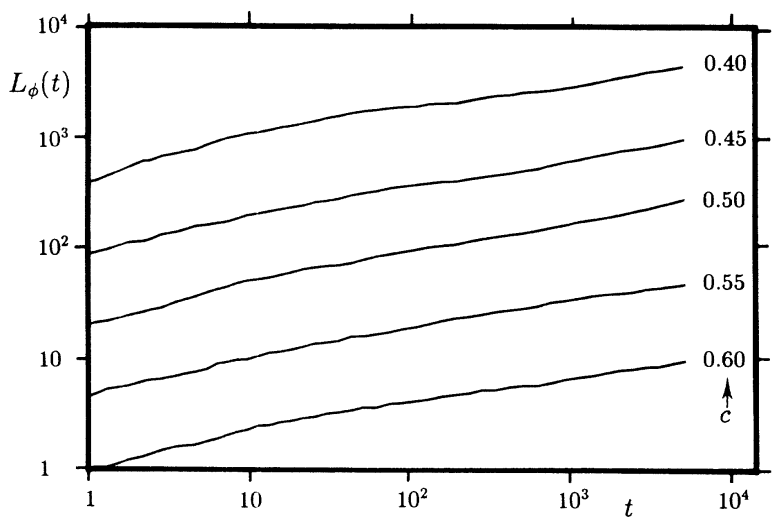

FIG. 6. Same as Fig. 4 for the length-scale measure $L_{\phi}(t)$, in Eq. (15), derived from the spin-order parameters of the $(2 \times 2)$ phase.

after some time and this peak moves towards larger values of $R$ as time elapses corresponding to the growth of the average linear domain size. By introducing the time-dependent scaling variable, $x=R(t) / \bar{R}(t)$, into the distribution function and by renormalizing in order to define the scaling function

$$
\tilde{P}(x)=\bar{R}(t) P(R, t)
$$

where $\int_{0}^{\infty} \tilde{P}(x) d x=1$, the inset in Fig. 8 demonstrates that such a scaling function exists and that scaling is fulfilled by the present set of data for $t \geq 10^{3} \mathrm{MCS} / \mathrm{s}$. Since the present set of data is rather limited and subject to considerable statistical uncertainty, these findings should be considered only as suggestive evidence for dynamical scaling.

\section{CONCLUSIONS}

In this paper we have investigated the nonequilibrium ordering and phase separation processes in a twodimensional diluted Ising model. The model has an equi-

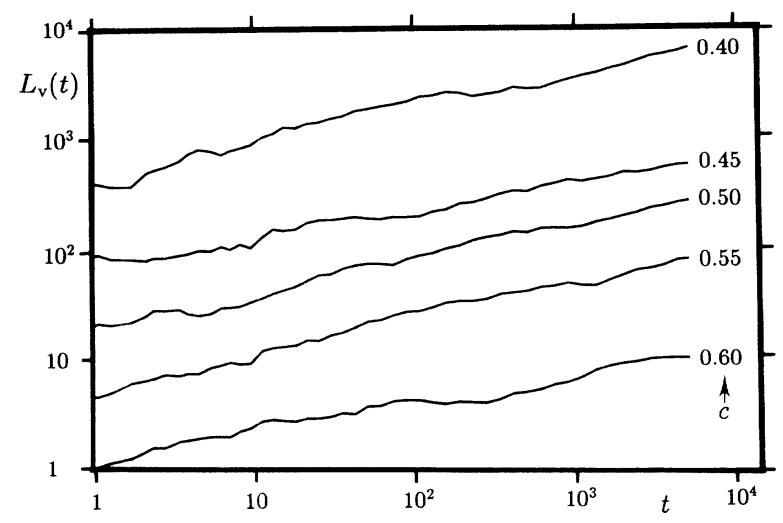

FIG. 7. Same as Fig. 4 for the length-scale measure, $L_{v}(t)$ in Eq. (16), derived from the vacancy order parameter. 


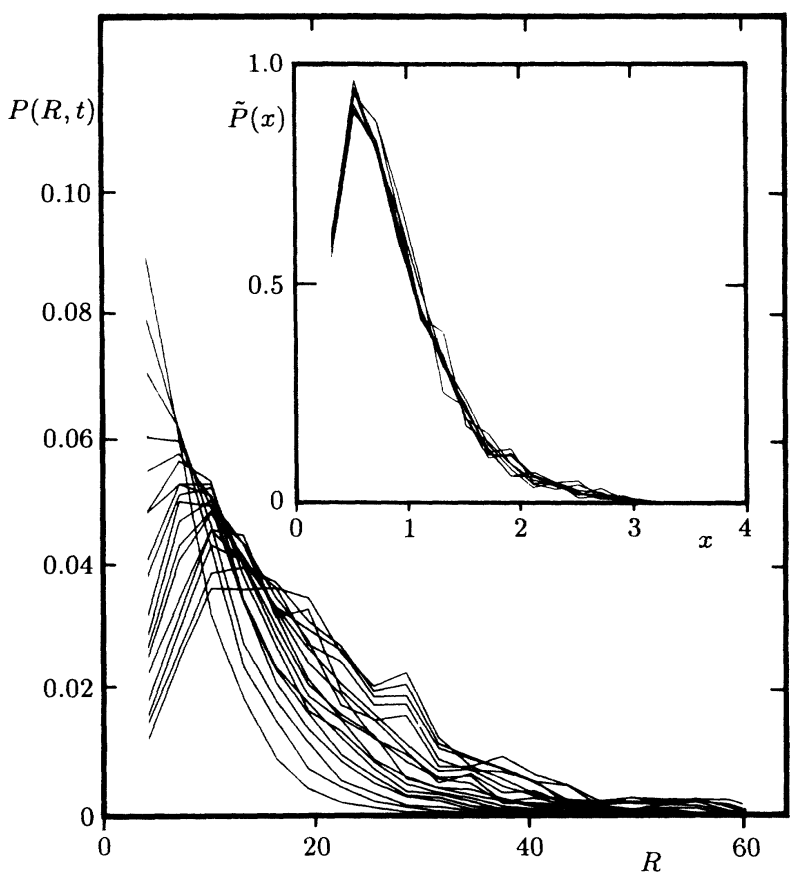

FIG. 8. Domain-size distribution function, $P(R, t)$, for $(2 \times 2)$ domains in the case of quenches to a temperature $k_{B} T / J=0.25$ for a vacancy concentration $c=0.60$. The data refer to domains of the $(2 \times 2)$ phase, cf. Fig. 3, obtained from Monte Carlo simulations on a lattice with $200 \times 200$ sites. A maximum in $P(R, t)$ develops and moves to the right as time elapses. Results are given for times $t=90,159,234,344$, $418,509,619,752,916,1115,1357,1653,2013,2449,2969$, 3589,4332 , and $5225 \mathrm{MCS} / \mathrm{s}$. The inset shows the corresponding dynamic scaling function, $\tilde{P}(x)$ in Eq. (18), where $x=R(t) / \bar{R}(t)$ is the scaling variable and $t \geq 1357 \mathrm{MCS} / \mathrm{s}$.

librium phase diagram, which at low temperatures contains regions of coexistence of phases that have different densities, cf. Fig. 2, specifically coexistence between $(2 \times 1)$ and $(2 \times 2)$ phases, and $(2 \times 2)$ and a gas phase. The order parameter is not conserved as the system evolves towards equilibrium. Furthermore, the ordered phases that are nucleated after the quench are fourfold degenerate. Hence we are considering a fairly complex pattern-formation phenomenon, which implies spin ordering, phase separation of the spin-ordered phases, as well as competition (domain growth) between the different thermodynamically degenerate domains of the individual spin-ordered phases. A further complication arises in the region of coexistence of the $(2 \times 1)$ and $(2 \times 2)$ phases, since these phases-although of very different densities-are energetically degenerate locally. Hence transient behavior may arise due to local competition between the two types of ordering. This competition will be governed by the local density, which in turn is controlled by long-range-diffusion processes.

Our general finding is that the ordering and separation processes, after quenches to either of the coexistence regions, are described by an algebraic growth law, Eq. (17).
Different measures of the growth lead to the same growth law and dynamical scaling seems to hold. The value of the growth exponent, $n \simeq 0.30 \pm 0.05$, is slightly below but not inconsistent with the value $n=\frac{1}{3}$ predicted by the Lifshitz-Slyozov theory ${ }^{26}$ for spinodal decomposition and phase separation in systems with a conserved order parameter and for tricritical quenching. It is not obvious that this theory should apply to the present case. Firstly, the Lifshitz-Slyozov theory accounts for two-component systems (e.g., double-degenerate ordering). Secondly, in our system the order parameter is not conserved. It has been argued, ${ }^{21,22}$ however, that the Lifshitz-Slyozov growth law may also apply to nonconserved systems, provided that the phase separation involves phases of different densities. In that case longrange diffusion controls the growth and the basic assumption of the Lifshitz-Slyozov theory is fulfilled. The results of the present work give further support to this argument. Additional numerical evidence of a similar nature is based on a study of the ordering processes in a diluted planar rotor model with competition between herringbone and pinwheel phases. ${ }^{39}$ This planar rotor model, which is a model of mixtures of, e.g., $\mathrm{N}_{2}$ (or $\mathrm{CO}$ ) and rare gases physisorbed on graphite, at dilution $\frac{1}{4}$ is in fact very similar to the model in Eq. (1) at $c=0.50$, since both of them in that case in equilibrium support a homogeneously ordered phase, a stoichiometric compound of $(2 \times 2)$ symmetry. Despite this fact, quenches of either model lead to effective nonequilibrium phase separation of the two phases, which are stable at the two sides of the stoichiometric composition. Hence, we do not observe formation of a homogeneous $(2 \times 2)$ phase at $c=0.50$ during the time course of the quenches reported in Fig. 3.

Systems of chemisorbed oxygen on W(110) surfaces are realizations of the ordering symmetries of the Ising model studied here. Obviously, due to higher-order and longerranged interactions the phase diagram ${ }^{34}$ of $\mathrm{O} / \mathrm{W}(110)$ is more involved than the one in Fig. 2. However, at subhalf-monolayer coverages $\mathrm{O} / \mathrm{W}(110)$ is found to phase separate into a gas phase and $\mathbf{a}(2 \times 1)$ phase, and for super-half-monolayer coverages into coexistence between a $(2 \times 1)$ and a $(2 \times 2)$ phase. The kinetic exponent values found in the two regimes are found by low-energy electron-diffraction (LEED) techniques ${ }^{34}$ to be $n \simeq 0.28$ and $n \simeq 0.2$, respectively. Recently, Tringides ${ }^{40}$ performed a LEED study of the phase-separation kinetics of the $\mathrm{O} / \mathrm{W}(110)-p(2 \times 1)+p(2 \times 2)$ system and found a larger exponent value $n \simeq 0.31 \pm 0.03$. This exponent value is probably more reliable than the earlier reported value $(0.2)$ since Tringides ${ }^{40}$ in his study for the lengthscale measure used the inverse second moment rather than the peak intensity of the scattering function. Furthermore, in this study the surface was carefully checked for impurities by Auger spectroscopy. Hence our theoretical finding is consistent with the most reliable experimental measurements. The tendency to find experimental exponent values on the low side of $\frac{1}{3}$ and in particular a lower effective exponent value in the coexistence region 
of the two ordered phases is consistent with the findings of the present study. Finally, it should be mentioned that the pioneering computer-simulation study ${ }^{41}$ within kinetics of ordering on surfaces was in fact devoted to modelling $\mathrm{O} / \mathrm{W}(110)$ at $c=0.25$ by using a lattice-gas model with a range of interaction extended to the third-nearest neighbors on a square lattice. A very slow kinetics was reported as described by an effective growth exponent value $n \simeq 0.15$. Recent work ${ }^{35,36}$ on the model studied in this paper, but at much lower vacancy concentrations, also led to a very slow (possibly logarithmic) growth behavior. Hence it appears that very slow kinetics may arise in certain parts of the phase diagram with leads to very low values of the effective growth exponent. Further work is needed to clarify whether or not this is a real asymptotic effect or just a transient or low-temperature crossover effect.

In closing we wish to remark that the complicated and slow growth behavior of competing and phase-separating domains of different density is likely to be a rather widespread phenomenon in condensed matter systems. A very timely example is that of oxygen ordering in the $\mathrm{CuO}$ basal planes of high- $T_{\mathrm{c}}$ superconductors of the $\mathrm{YBa}_{2} \mathrm{Cu}_{3} \mathrm{O}_{7-\delta}$ type. This two-dimensional ordering problem, which is related to the superconducting properties of the material, is controlled by long-range oxygen diffusion and it involves ordered phases of different densities, specifically the orthorhombic I and II phases. ${ }^{42} \mathrm{~A}$ recent simulation study ${ }^{43}$ of this problem indeed shows that the overall ordering process for the oxygen atoms is indeed very slow.

\section{ACKNOWLEDGMENTS}

This work was supported by the Danish Natural Science Research Council under Grant Nos. J.nr. 5.21.99.72 and 11-7785 and by the Danish Technical Research Council under Grant No. J.nr. 16-4296.K. Funding for supercomputer time for the present work was provided via a special grant from the Danish Natural Science Research Council and UNI.C (J.nr. 11-8020) which gave access to the Amdahl VP1100 vectorprocessor at UNI.C. We wish to thank Michael Tringides for useful comments of our work and for making his experimental results available to us before publication.
${ }^{1}$ J. D. Gunton, M. San Miguel, and P.S. Sahni, in Phase Transitions and Critical Phenomena, edited by C. Domb and J. L. Lebowitz (Academic, New York, 1983), Vol. 8, p. 267.

${ }^{2}$ H. Furukawa, Adv. Phys. 34, 703 (1985).

${ }^{3} \mathrm{O}$. G. Mouritsen, in Kinetics of Ordering and Growth at Surfaces, edited by M. G. Lagally (Plenum, New York, in press).

${ }^{4} \mathrm{~K}$. Binder, in Materials Science and Technology, Vol. 5: Phase Transformations in Materials, edited by P. Haasen (VCH Verlagsgesellschaft, Berlin, in press).

${ }^{5}$ For a recent overview of the field of ordering dynamics, see the selection of papers in Dynamics of Ordering Processes in Condensed Matter, edited by S. Komura and H. Furukawa (Plenum, New York, 1988).

${ }^{6}$ P. C. Hohenberg and B. I. Halperin, Rev. Mod. Phys. 49, 435 (1977).

${ }^{7}$ S. Katano and M. lizumi, Phys. Rev. Lett. 52, 835 (1984).

${ }^{8}$ B.D. Gaulin, S. Spooner, and Y. Morii, Phys. Rev. Lett. 59, 668 (1987).

${ }^{9}$ D. Roux and C. M. Knobler, Phys. Rev. Lett. 60, 373 (1988).

${ }^{10}$ P. Wiltzius, F. S. Bates, and W. R. Heffner, Phys. Rev. Lett. 60, 1538 (1988).

${ }^{11}$ J. G. Amar, F. E. Sullivan, and R. D. Mountain, Phys. Rev. B 37, 196 (1988).

${ }^{12}$ A. Milchev, D. W. Heermann, and K. Binder, Acta Metall. 36, 377 (1988).

${ }^{13}$ R. Toral, A. Chakrabarti, and J. D. Gunton, Phys. Rev. Lett. 60, 2311 (1988).

${ }^{14}$ T. M. Rogers, K. R. Elder, and R. C. Desai, Phys. Rev. B 37, 9638 (1988).

${ }^{15}$ Y. Oono and S. Puri, Phys. Rev. A 38, 434 (1988); S. Puri and Y. Oono, ibid. 38, 1542 (1988).

${ }^{16}$ T. M. Rogers and R. C. Desai, Phys. Rev. B 39, 11956
(1989).

${ }^{17}$ C. Roland and M. Grant, Phys. Rev. B 39, 11971 (1989).

${ }^{18}$ Q. Zheng and J. D. Gunton, Phys. Rev. A 39, 4848 (1989).

${ }^{19}$ E. T. Gawlinski, J. D. Gunton, and J. Viñals, Phys. Rev. B 39, 7266 (1989).

${ }^{20}$ H. C. Fogedby and O. G. Mouritsen, Phys. Rev. B 37, 5962 (1988).

${ }^{21}$ A. Sadiq and K. Binder, J. Stat. Phys. 35, 517 (1984).

${ }^{22}$ W. W. Mullins and J. Viñals, Acta. Metall. 37, 991 (1989).

${ }^{23}$ M. San Miguel, J. D. Gunton, G. Dee, and P. S. Sahni, Phys. Rev. B 23, 2334 (1981).

${ }^{24}$ T. Ohta, K. Kawasaki, A. Sato, and Y. Enomoto, Phys. Lett. A 126, 93 (1987).

${ }^{25}$ A. Chakrabarti, J. B. Collins, and J.D. Gunton, Phys. Rev. B 38, 6894 (1988).

${ }^{26}$ I. M. Lifshitz and V. V. Slyozov, J. Phys. Chem. Solids 19, 35 (1961).

${ }^{27}$ P. W. Voorhees, J. Stat. Phys. 38, 231 (1985).

${ }^{28}$ D. A. Huse, Phys. Rev. B 34, 7845 (1986).

${ }^{29}$ C. Yeung, Phys. Rev. B 39, 9652 (1989).

${ }^{30} \mathrm{~K}$. Heinz, in Kinetics of Interface Reactions, edited by M. Grunze and H. J. Kreuzer (Springer-Verlag, New York, 1987), p. 207.

${ }^{31}$ J.-K. Zuo, G.-C. Wang, and T.-M. Lu, Phys. Rev. B 39, 9432 (1989).

${ }^{32}$ M. C. Tringides, P. K. Wu, and M. G. Lagally, Phys. Rev. Lett. 59, 315 (1987).

${ }^{33}$ J.-K. Zuo, G.-C. Wang, and T.-M. Lu, Phys. Rev. Lett. 60, 1053 (1988).

${ }^{34}$ P. K. Wu, M. C. Tringides, and M. G. Lagally, Phys. Rev. B 39, 7595 (1989).

${ }^{35}$ O. G. Mouritsen and P. J. Shah, Phys. Rev. B 40, 11445 (1989).

${ }^{36}$ P. J. Shah and O. G. Mouritsen, Phys. Rev. B 41, 7003 (1990). 
${ }^{37}$ A. Milchev, K. Binder, and D.W. Heermann, Z. Phys. B 61, 521 (1986).

${ }^{38} \mathrm{~K}$. Binder and D. Stauffer, Phys. Rev. Lett. 33, 1006 (1974).

${ }^{39}$ O.G. Mouritsen, Phys. Rev. B 32, 1632 (1985).

${ }^{40}$ M.C. Tringides (unpublished).

${ }^{41}$ P.S. Sahni and J.D. Gunton, Phys. Rev. Lett. 47, 1754
(1981).

42 J.D. Jorgensen, M.A. Beno, D.G. Hinks, L. Soderholm, K.J. Volin, R.L. Hitterman, J.D. Grace, I.K. Schuller, C.V. Segre, K. Zhang, and M.S. Kleefisch, Phys. Rev. B 36, 3608 (1987).

${ }^{43}$ J.V. Andersen, H. Bohr, and O.G. Mouritsen, Phys. Rev. B 42, 283 (1990). 

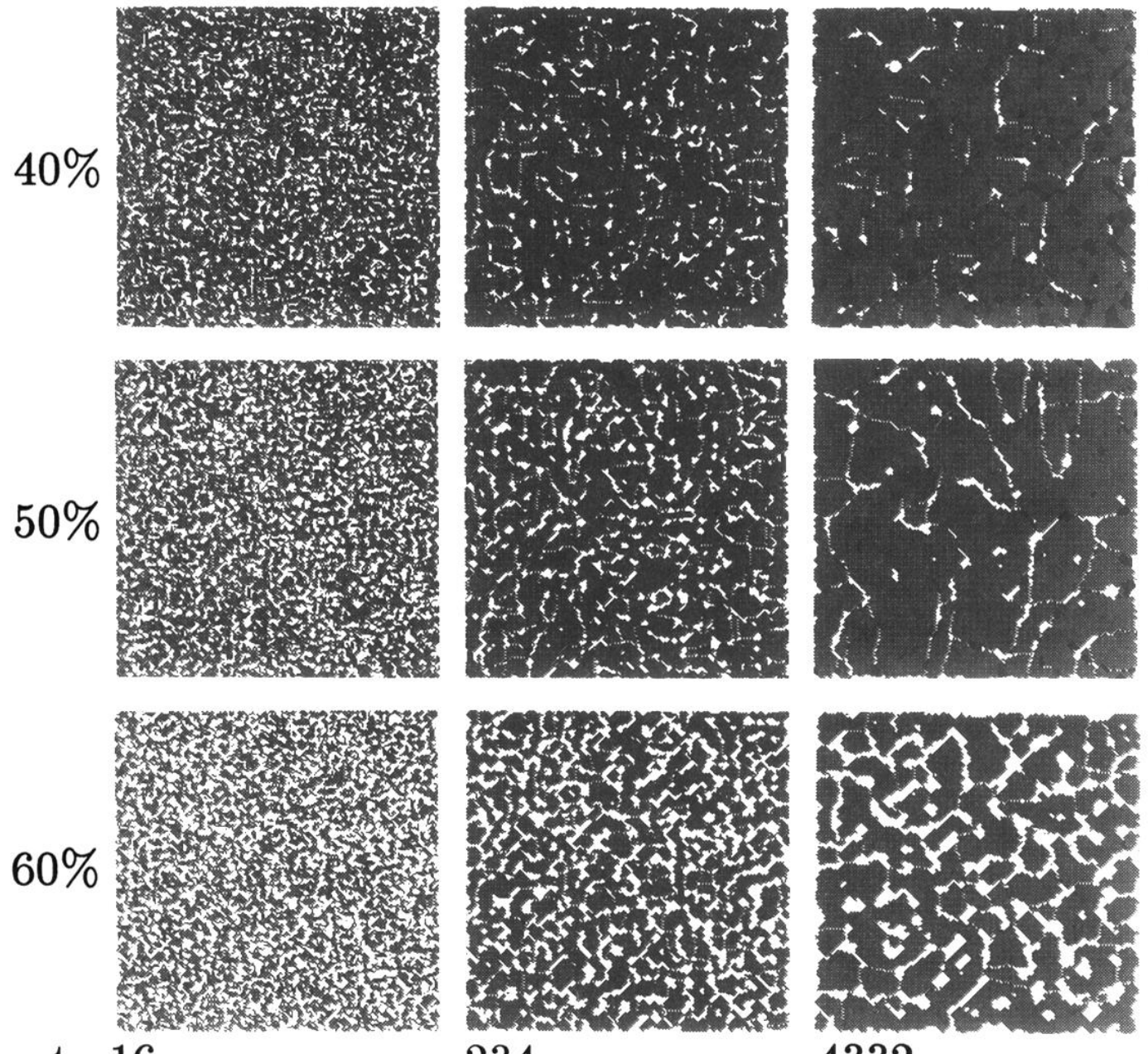

$\mathrm{t}=16$ 234

4332

FIG. 3. Snapshots of typical microconfigurations at three different concentrations as they evolve in time $t$ (in units of $\mathrm{MCS} / \mathrm{s})$ after quenches to a temperature $k_{B} T / J=0.25$. Vacancies are denoted by blanks, $(2 \times 1)$ ordering is indicated in black, and $(2 \times 2)$ ordering appears as grey. 